\title{
Dietary Patterns and the Risk of Inflammatory Bowel Disease: Findings from a Case-Control Study
}

Reema F. Tayyem¹,2,3, Tamara R. Qalqili³, Rawan Ajeen ${ }^{4}$ and Yaser M. Rayyan ${ }^{3}$

\author{
${ }^{1}$ Department of Human Nutrition, College of Health Sciences, QU Health, Qatar University, Doha, Qatar \\ Biomedical and Pharmaceutical Research Unit, QU Health, Qatar University, Doha, Qatar \\ ${ }^{3}$ The University of Jordan, Amman, Jordan; \\ ${ }^{4}$ University of North Carolina at ChapelHill, Chapel Hill, NC, USA
}

\section{Introduction}

Scientific evidence shows that dietary patterns are associated with the risk of IBD, particularly among unhealthy and Western dietary patterns. However, Western dietary patterns are not exclusive to Western countries, as Jordanians are steadily moving towards a Western lifestyle, which includes an increased consumption of processed foods. This study aims to investigate the association between dietary patterns and the risk factors for IBD cases among Jordanian adults.

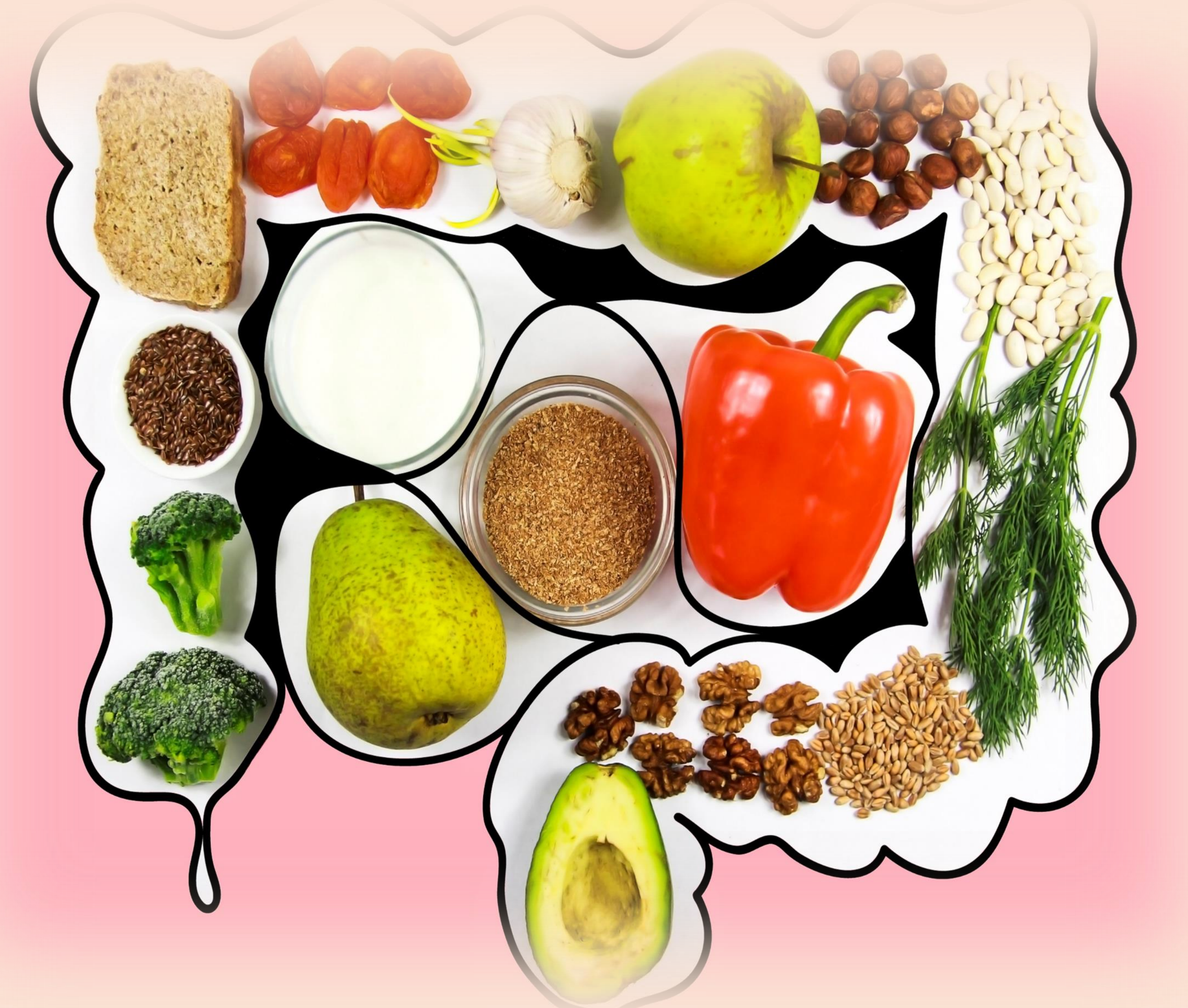

\section{Methods}

This case-control study was conducted between November 2018 and December 2019 in the largest three hospitals in Jordan. Three hundred and thirty-five Jordanian adults aged between 18-68 years were enrolled in this study: one hundred and eighty-five IBD patients who were recently diagnosed with IBD ( $n=100$ for ulcerative colitis (UC) and $n=\mathbf{8 5}$ for Crohn's disease (CD)) and 150 IBD-free controls.

Participants were matched based on age and marital status. In addition, dietary data was collected from all participants using a validated food frequency questionnaire. Factor analysis and principal component analysis were used to determine the dietary patterns. Odds ratios (OR) and their $95 \%$ confidence interval $(\mathrm{Cl})$ were calculated using a multinomial logistic regression model.

\section{Findings}

Two dietary patterns were identified among the study participants: high-vegetable and high-protein dietary patterns. There was a significantly higher risk of IBD with high-protein intake. In contrast, the high-vegetable dietary pattern shows a significant protective effect on IBD in the third and fourth quartiles in all the models.

Association between IBD risk and dietary patterns among the IBD ( $n=185)$ and controls $(n=150)$

\begin{tabular}{|c|c|c|c|c|}
\hline Variables & Q1 & Q2 & Q3 & Q4 \\
\hline \multicolumn{5}{|c|}{ Crude $\mathrm{OR}$ and $\mathrm{Cl}^{\#}$} \\
\hline High-Vegetables & 1 & $0.648(0.339-1.24)$ & $0.136(0.068-0.271)$ & $0.126(0.064-0.248)$ \\
\hline Controls/Cases & $21 / 68$ & $20 / 60$ & $51 / 32$ & $59 / 27$ \\
\hline High-Protein & 1 & $1.130(0.572-2.235)$ & $2.196(1.046-4.610)$ & $4.391(2.67-8.506)$ \\
\hline Controls/Cases & $43 / 40$ & $57 / 27$ & $25 / 46$ & $25 / 52$ \\
\hline \multicolumn{5}{|c|}{ Adjusted $\mathrm{OR}$ and $\mathrm{Cl}$ “Adjusted for age and gender ** } \\
\hline High-Vegetables & 1 & $0.669(0.241-1.2853)$ & $0.142(0.071-0.285)$ & $0.128(0.064-0.255)$ \\
\hline Controls/Cases & $21 / 68$ & $20 / 60$ & $51 / 32$ & $59 / 27$ \\
\hline High-Protein & 1 & $1.091(0.549-2.165)$ & $2.216(1.146-4.210)$ & $4.215(2.166-8.204)$ \\
\hline Controls/Cases & $43 / 40$ & $57 / 27$ & $25 / 46$ & $25 / 52$ \\
\hline
\end{tabular}

Adjusted OR and CI "Adjusted for age, BMI, gender, physical activity, energy, marital status, education level, and smoking"

\begin{tabular}{lcccc} 
High-Vegetables & 1 & $0.664(0.336-1.2853)$ & $0.127(0.060-0.261)$ & $0.114(0.055-0.237)$ \\
\hline Controls/Cases & $21 / 68$ & $20 / 60$ & $51 / 32$ & $59 / 27$ \\
\hline High-Protein & 1 & $1.003(0.483-2.085)$ & $2.196(1.101-4.357)$ & $5.452(2.646-11.232)$ \\
\hline Controls/Cases & $43 / 40$ & $57 / 27$ & $25 / 46$ & $25 / 52$ \\
\hline
\end{tabular}

\section{Conclusion}

A high-vegetable dietary pattern may be protective against the risk of IBD, while a high-protein dietary pattern is associated with an increased risk of IBD among a group of the Jordanian population.

\section{Cited as:}

Tayyem, R.F.; Qalqili, T.R.; Ajeen, R.; Rayyan, Y.M. Dietary Patterns and the Risk of Inflammatory Bowel Disease: Findings from a CaseControl Study. Nutrients 2021,13, 1889 